\title{
Análisis y evaluación de los niveles de inclusión digital en Andalucía. Balance actual y retos de futuro
}

\author{
María Teresa Vera BALANZA \\ Universidad de Málaga \\ mvb@uma.es \\ María Purificación SuBIRES MANCERA \\ Universidad de Málaga \\ purificacion@uma.es
}

Recibido: 21/10/2012

Aceptado: 23/01/2013

\section{Resumen \\ A partir del análisis de la Encuesta sobre equipamiento y uso de tecnologías de la información y comu- nicación en los hogares (INE, 2012), proponemos la evaluación de los niveles de inclusión digital en Andalucía y estudiamos las variables más significativas que dibujan la denominada "brecha digital". \\ Palabras clave: Inclusión digital, brecha digital, TIC, Internet, Andalucía \\ Analysis and Evaluation of Digital Inclusion Levels in Andalusia. Current Assessment and Future Challenges}

\begin{abstract}
In this article, we analyse the Survey on the Equipment and Use of Information and Communication Technologies (ICT-H) in Households (INE, 2012) and propose the evaluation of digital inclusion levels in Andalusia. We study the most significant variables that draw the "digital divide".

Keywords: Digital inclusion, digital divide, ICT, Internet, Andalusia

\section{Referencia normalizada}

VERA BALANZA, María Teresa y SUBIRES MANCERA, María Purificación (2013): “Análisis y evaluación de los niveles de inclusión digital en Andalucía. Balance actual y retos de futuro". Estudios sobre el Mensaje Periodístico. Vol. 19, Núm. especial marzo, págs.: 511-521. Madrid, Servicio de Publicaciones de la Universidad Complutense.
\end{abstract}

Sumario: 1. Introducción. 2. Metodología. 3. Desarrollo. 4. Conclusiones. 5. Referencias bibliográficas

\section{Introducción}

Entendemos la inclusión digital como "la participación plena de todos los ciudadanos, en igualdad de condiciones, en la Sociedad del Conocimiento [...]" (Soto y Fernández 2004: 1). Desde la perspectiva opuesta, el antropólogo Scott S. Robinson alerta sobre el marcado cariz mercantilista en el impulso de las TIC en la sociedad y reafirma el papel de las políticas públicas (2005: 127) en "las garantías de privacidad y seguridad", la formación y promoción de software de código abierto o el compromiso presupuestario por parte de los gobiernos. López y Samek (2009) defienden el valor de la inclusión digital como derecho humano vinculado a las exigencias de la dignidad y la igualdad de los seres humanos, e instan a los poderes públicos a su reconocimiento. Este enfoque se vincula con las propias bases del estado moderno donde las condiciones y garantías aparecen entroncadas, en nuestro caso, a derechos relacionados con la información y la comunicación. Si el Estado liberal se sustentó sobre una 
serie de derechos políticos y civiles donde la libertad de expresión y de pensamiento adquirían un carácter fundamental, el Estado del Bienestar se articula sobre la universalización de los derechos económicos, sociales y culturales. Ahora, la Sociedadred ha de asegurar los derechos colectivos o de solidaridad mediante el estatuto jurídico sobre la vida humana derivado de las nuevas condiciones favorecidas por las tecnologías de la comunicación (Vasak, 1982). Estos derechos de cuarta generación, entienden que "la inclusión digital es un nuevo derecho humano procedente del nuevo entorno tecnológico que ha creado la red" (referencia a Gómez en López y Samek, 2009: 6); de manera equivalente, la exclusión digital deviene también en exclusión social. Así lo reconoció el Parlamento Europeo en 2001 y lo ha ratificado recientemente la ONU en sesión del pasado mes de julio.

Inicialmente, el origen de las políticas encaminadas al desarrollo de la Sociedad de la Información se sitúa en las propuestas realizadas en el año 1994 por el entonces vicepresidente de los EEUU, Al Gore, en la Asamblea de la Unión Internacional de las Telecomunicaciones, bajo la denominación de "Infraestructura Global de la Información", IGI (Agustín y Clavero, 2011: 257). Desde UNESCO el hito más destacado en relación con la inclusión digital es la celebración de la Cumbre Mundial de la Sociedad de la Información, celebrada en dos fases, Ginebra 2003 y Túnez 2005; es en la primera cita donde se acuñó el concepto de inclusión digital por parte de colectivos ciudadanos que impulsaron una campaña con el objetivo de promover los derechos de comunicación en la sociedad de la información; en la segunda, se aborda la medición de los efectos económicos y sociales de las TIC (Agustín y Clavero, 2011: 148).

En el caso de la Unión Europea, coincidentemente también en el año 1994 se data en el Informe Bangemann, sobre Europa y la sociedad global de la información, acerca de la creación de infraestructuras de información. La Comisión europea elabora además el Informe El camino de Europa hacia la Sociedad de la Información que se sustenta sobre tres puntos clave: la creación de un marco regulador, la puesta en marcha del servicio de redes y la generación de un espacio sociocultural para la innovación. Pero sin duda, uno de los más importantes hitos en esta secuencia lo constituye el Consejo Europeo de Lisboa, del año 2000, en el que se elabora la Estrategia que tiene como objetivo el establecimiento de indicadores y pautas de referencia cuantitativas y cualitativas para evaluar comparativamente a los estados miembros. El siguiente paso para el afianzamiento de la Sociedad de la Información en Europa será el programa eEurope, que se basa en "la implementación de medidas concretas sobre telecomunicaciones, el establecimiento de un marco jurídico para el comercio electrónico y el apoyo a la industria y a la I+D”, que cuenta con planes de acción de carácter anual (Agustín y Clavero, 2011: 155), de los que se ejecutaron los correspondientes a 2002 y a 2005. Tras ellos, el Plan i2010 La Sociedad de la información y los medios de comunicación al servicio del crecimiento y del empleo (20062010) ya "sustituye los indicadores de infraestructuras por indicadores de impacto" (Agustín y Clavero, 2011, 155). En abril de 2010 se aprueba la Declaración de Granada para la nueva Agenda Digital Europea, a través de la que se pretende reforzar la alfabetización digital, las capacidades y destrezas y la inclusión digital, donde se incardina la Estrategia Europa 2020 (UE, 2010: web) para un crecimiento inteligente, sostenible e integrador. 
En el caso de España, el primero de los planes puestos en marcha para el fomento de la Sociedad de la Información, fue el programa Info XXI: La Sociedad de la Información para todos (2000 a 2003) entre cuyas iniciativas incluía la habilitación de Puntos de Acceso Público a Internet.

Hacia mitad del período, a finales de 2002, como resultado del informe sobre la situación de la Sociedad de la Información, surge el proyecto España.es, con carácter propulsor. Este segundo plan, que tiene una duración de dos años (2004-2005), cuenta con seis líneas maestras: tres verticales -Administración Electrónica, Educación y Pymes- y tres horizontales -accesibilidad y formación, contenidos digitales y comunicación- (Caridad et. al. 2006: 18). Luego siguió el plan Ingenio 2010, presentado en junio de 2005, y que incluía cuatro programas: Cenit, Consolider, Euroingenio y Plan Avanz@ "para alcanzar la media europea en los indicadores de la Sociedad de la Información".

El Plan Avanza, desarrollado durante el período 2006-2010, actuaba en cinco áreas: Hogar e Inclusión de Ciudadanos, Competitividad e Innovación, Educación en la Era Digital, Servicios Públicos Digitales y Contexto Digital (2006: 8). La continuación fue el Plan Avanza2 (2009-2015) donde aboga por el uso sostenible de las TIC. La última actuación realizada por el ejecutivo español es la promoción de un proceso de consulta pública para la mejora de la propuesta de Agenda Digital para España (http://www.agendadigital.gob.es/), cuyo plazo para opinar, debatir, y lanzar nuevas iniciativas finalizó en septiembre de 2012.

En 2002 en Andalucía se pone en marcha el Plan I@1andalus de Iniciativas Estratégicas para el Desarrollo de la Sociedad de la Información, que actuaba esencialmente sobre la creación de infraestructuras, la de servicios electrónicos de calidad y la concienciación de la ciudadanía sobre la utilidad de las TIC. Uno de los principales hitos derivados de este plan fue la puesta en marcha del Programa Guadalinfo, por el que se creó, entre 2002 y 2004, la Red de Centros de Acceso Público a Internet por banda ancha con el objetivo estratégico de reducir la brecha digital entre quienes tienen acceso o no a las nuevas tecnologías digitales, asegurando unas instalaciones y fomentando la alfabetización digital, especialmente en los municipios menores de 10.000 habitantes. En marzo de 2003 se promulga el Decreto de Medidas de Impulso a la Sociedad del Conocimiento en Andalucía, acompañado de sendos decretos (137/2004 y 7/2004) que incentivan la adquisición de equipos informáticos y bonificaciones de las tarifas de conexión a Internet en el ámbito doméstico.

Seguidamente, el Plan Andalucía Sociedad de la Información (2007-2010) se estructura sobre cuatro focos: Comunidad digital, Empresas para la nueva economía, Administración inteligente e Infraestructura y contexto digital. Es en el primer ámbito donde se promueve la inclusión digital, teniendo en cuenta que existen diferencias de penetración en el uso de las TIC que se originan en factores culturales, económicos y geográficos, con especial impacto en colectivos tradicionalmente en riesgo de exclusión, que en la práctica equivalen, preferentemente, a las mujeres, las personas mayores y las que se encuentran en situación de dependencia, la adaptación de servicios TIC a personas con discapacidades, así como acercar los conocimientos y el uso de las nuevas tecnologías a la población en situación, entorno o zonas desfavorecidas. 


\section{Metodología}

Teniendo en cuenta la afanosa búsqueda de una métrica y de indicadores comunes para dimensionar la sociedad de la comunicación, actualmente contamos con datos armonizados que permiten el análisis comparado pero revela, asimismo, los límites de las metodologías exclusivamente cuantitativas.

Así, la situación vigente es consecuente con las políticas desarrolladas en tanto que enfocadas hacia la extensión del equipamiento y los usos. Tras esta fase, descrita y evaluada, se imponen, lógicamente, las acciones específicas sobre áreas deficitarias.

Contamos pues con series estadísticas procedentes de fuentes oficiales, especialmente las Encuesta sobre equipamiento y uso de tecnologías de la información y comunicación en los hogares que se viene elaborando con periodicidad anual desde 2002, que recoge información sobre los diversos productos de tecnologías de información y comunicación de los hogares españoles así como los usos que se hacen de estos productos, de Internet y del comercio electrónico. Consecuentemente con los usos y los perfiles, se dedica una atención especial a los usos por parte de la infancia; esta particularidad señala -en negativo- un sesgo diferencial que deviene en brecha: la edad (al no incluir en la encuesta a las personas mayores de 74 años). En esta ocasión utilizaremos los datos de 2012 hechos públicos recientemente, y desagregados por regiones.

Hemos procedido al análisis comparado que nos permita cuantificar la magnitud de las brechas o diferenciales en función de diversas variables, entendiendo que éstas actúan siempre de manera combinada pero con efectos exponencialmente multiplicados.

\section{Desarrollo}

a) Comparativa por Comunidades Autónomas

Analizando los resultados de la última Encuesta sobre Equipamiento y Uso de Tecnologías de la Información y Comunicación en los hogares (INE, 2012: web), podemos destacar, en primer lugar, que tanto en disponibilidad de equipamiento e infraestructuras, como en uso de las TIC, Andalucía se sitúa muy ligeramente por debajo de la media nacional.

Tabla 1. Fuente: INE (2012)

\begin{tabular}{|c|c|c|c|}
\hline \multicolumn{4}{|c|}{ Equipamiento e infraestructuras y usos TIC. España y Andalucía, 2012 (\%) } \\
\hline & Variable & Total nacional & Andalucía \\
\hline \multirow{2}{*}{$\begin{array}{c}\text { Equipamiento e } \\
\text { infraestructuras. } \\
\text { Viviendas } \\
\text { con... }\end{array}$} & Algún tipo de ordenador & 73,9 & 73,2 \\
\cline { 2 - 4 } & Acceso a Internet & 67,9 & 65,2 \\
\cline { 2 - 4 } & Conexión de Banda Ancha & 66,7 & 63,1 \\
\hline \multirow{3}{*}{$\begin{array}{c}\text { Usos TIC. } \\
\text { Personas que, en } \\
\text { los últimos tres } \\
\text { meses,... }\end{array}$} & Teléfono móvil & 95,9 & 95,3 \\
\cline { 2 - 4 } & Han utilizado el ordenador & 72,2 & 68,4 \\
\cline { 2 - 4 } & Han utilizado Internet & 69,8 & 67,0 \\
\cline { 2 - 4 } & Han compradilizado Internet al menos una través de Internet & 65,0 & 61,4 \\
\cline { 2 - 4 } & Han utilizado el teléfono móvil & 22,3 & 17,9 \\
\hline
\end{tabular}


Si comparamos por Comunidades Autónomas, con datos referidos a viviendas, Andalucía ocupa el noveno puesto en posesión de ordenador, el undécimo en disponibilidad de acceso a Internet y el duodécimo, tanto en conexión de banda ancha, como en telefonía móvil. Si nos centramos en el uso de las TIC en los tres últimos meses, Andalucía es la decimotercera en cuanto a uso del ordenador, la décima respecto a utilización de Internet y la undécima en cuanto a su empleo al menos una vez a la semana. Además, se ubica en el decimotercer puesto en compras a través de Internet y en el séptimo en el caso de la utilización del teléfono móvil.

Estos datos nos demuestran que Andalucía se sitúa en una posición intermedia-a la baja, tanto en equipamiento como en uso, respecto al resto de comunidades autónomas, lo que nos advierte de una ya bien conocida brecha territorial en el acceso a las TIC dentro de nuestro país, pese a los esfuerzos realizados tanto por parte de la administración central como de las distintas administraciones autonómicas en pro de la inclusión digital.

b) Evolución en Andalucía (2004-2012)

Tabla 2. Fuente: INE (2012) y elaboración propia

\begin{tabular}{|c|c|c|c|c|c|c|c|c|c|c|}
\hline \multicolumn{11}{|c|}{$\begin{array}{l}\text { Evolución de equipamiento e infraestructuras y usos TIC. } \\
\text { España y Andalucía, 2004- } 2012(\%)\end{array}$} \\
\hline & & 2004 & 2005 & 2006 & 2007 & 2008 & 2009 & 2010 & 2011 & 2012 \\
\hline \multicolumn{11}{|c|}{ Viviendas con.. } \\
\hline \multirow[t]{3}{*}{ Ordenador } & España & 52,3 & 54,9 & 57,2 & 60,4 & 63,6 & 66,3 & 68,7 & 71,5 & 73,9 \\
\hline & Andalucía & 47,9 & 48,7 & 52,4 & 56,9 & 59,4 & 62,3 & 66,6 & 69,6 & 73,2 \\
\hline & Dif. A-E & $-4,4$ & $-6,2$ & $-4,8$ & $-3,5$ & $-4,2$ & -4 & $-2,1$ & $-1,9$ & $-0,7$ \\
\hline \multirow{3}{*}{$\begin{array}{l}\text { Acceso a } \\
\text { Internet }\end{array}$} & España & 33,6 & 35,5 & 39,1 & 44,6 & 51 & 54 & 59,1 & 63,9 & 67,9 \\
\hline & Andalucía & 26 & 28,1 & 31,7 & 38,9 & 43,7 & 48,4 & 54,5 & 59,5 & 65,2 \\
\hline & Dif. A-E & $-7,6$ & $-7,4$ & $-7,4$ & $-5,7$ & $-7,3$ & $-5,6$ & $-4,6$ & $-4,4$ & $-2,7$ \\
\hline \multirow{3}{*}{$\begin{array}{c}\text { Conexión } \\
\text { de Banda } \\
\text { Ancha }\end{array}$} & España & 14,7 & 21,1 & 29,3 & 39,2 & 44,6 & & 57,4 & 61,9 & 66,7 \\
\hline & Andalucía & 11,5 & 17,1 & 24,2 & 35 & 38,9 & 46,3 & 52,9 & 56,2 & 63,1 \\
\hline & Dif. A-E & $-3,2$ & -4 & $-5,1$ & $-4,2$ & $-5,7$ & -5 & $-4,5$ & $-5,7$ & $-3,6$ \\
\hline \multirow{3}{*}{$\begin{array}{c}\text { Teléfono } \\
\text { móvil }\end{array}$} & España & 82,4 & 85,3 & 88,1 & 90,9 & 92,1 & 93,5 & 94,6 & 95,1 & 95,9 \\
\hline & Andalucía & 80,7 & 82,6 & 86,2 & 89,4 & 90,1 & 92,3 & 93,6 & 93,8 & 95,3 \\
\hline & Dif. A-E & $-1,7$ & $-2,7$ & $-1,9$ & $-1,5$ & -2 & $-1,2$ & -1 & $-1,3$ & $-0,6$ \\
\hline \multicolumn{11}{|c|}{$\begin{array}{l}\text { Personas que en los } 3 \\
\text { últimos meses ... }\end{array}$} \\
\hline \multirow{3}{*}{$\begin{array}{c}\text { Han } \\
\text { utilizado el } \\
\text { ordenador }\end{array}$} & España & 49 & 52,1 & 54 & 57,2 & 61 & 63,2 & 67,4 & 69,3 & 72,2 \\
\hline & Andalucía & 42,3 & 43,7 & 47,3 & 52,8 & 55,5 & 59,2 & 63,2 & 64,8 & 68,4 \\
\hline & Dif. A-E & $-6,7$ & $-8,4$ & $-6,7$ & $-4,4$ & $-5,5$ & -4 & $-4,2$ & $-4,5$ & $-3,8$ \\
\hline \multirow{3}{*}{$\begin{array}{c}\text { Han } \\
\text { utilizado } \\
\text { Internet }\end{array}$} & España & 40,4 & 44,4 & 47,9 & 52 & 56,7 & 59,8 & 64,2 & 67,1 & 69,8 \\
\hline & Andalucía & 32,5 & 36,9 & 42 & 47 & 50,7 & 54,8 & 59,2 & 62,4 & 67 \\
\hline & Dif. A-E & $-7,9$ & $-7,5$ & $-5,9$ & -5 & -6 & -5 & -5 & $-4,7$ & $-2,8$ \\
\hline \multirow{3}{*}{$\begin{array}{c}\text { Han } \\
\text { comprado } \\
\text { a través de } \\
\text { Internet }\end{array}$} & España & 5,5 & 8 , & 10,1 & 13 & 13,3 & 15,7 & 17,4 & 18,9 & 22,3 \\
\hline & Andalucía & 3,3 & 5,2 & 6,3 & 9,5 & 9,7 & 11,5 & 13,4 & 14,7 & 17,9 \\
\hline & Dif. A-E & $-2,2$ & $-2,9$ & $-3,8$ & $-3,5$ & $-3,6$ & $-4,2$ & -4 & $-4,2$ & $-4,4$ \\
\hline
\end{tabular}

Tras el análisis de los porcentajes correspondientes al total de España y a la Comunidad Autónoma Andaluza, advertimos que desde el año 2004 hasta el 2012 -con 
la única excepción de las compras a través de Internet-, se ha producido una disminución gradual de la diferencia entre ambos. El acercamiento más evidente se encuentra en la disponibilidad de ordenador y de acceso a Internet, así como en uso de la Red, durante el año 2012.

En el actual contexto de crisis económica, estos datos pueden reflejar el interés de la población por disponer de unos recursos que les faciliten el acceso a la información y a la educación, la búsqueda de empleo y el ocio, además de mostrar, de algún modo, los resultados a largo plazo de las políticas públicas para la inclusión digital llevadas a cabo. A ello hemos de sumar la estabilización de los costes de los equipos y la multiplicación de la oferta de acceso a Internet, que permite a los usuarios disponer de unos precios más competitivos.

En el siguiente gráfico apreciamos la evolución experimentada en estos últimos años en relación con la disponibilidad de ordenador e Internet, en el conjunto de España y en Andalucía, en el marco de los respectivos planes materializados en dicha comunidad. En ambos casos, observamos una tendencia a que las líneas converjan entre sí, especialmente en el del ordenador. Si disponer de equipamiento supone el primer paso para la inclusión digital, aquí podemos ver como Andalucía -pese a la situación económica- no se baja del carro y va acercándose progresivamente a la media española.

Comparativa ordenador e Internet (España y Andalucia)

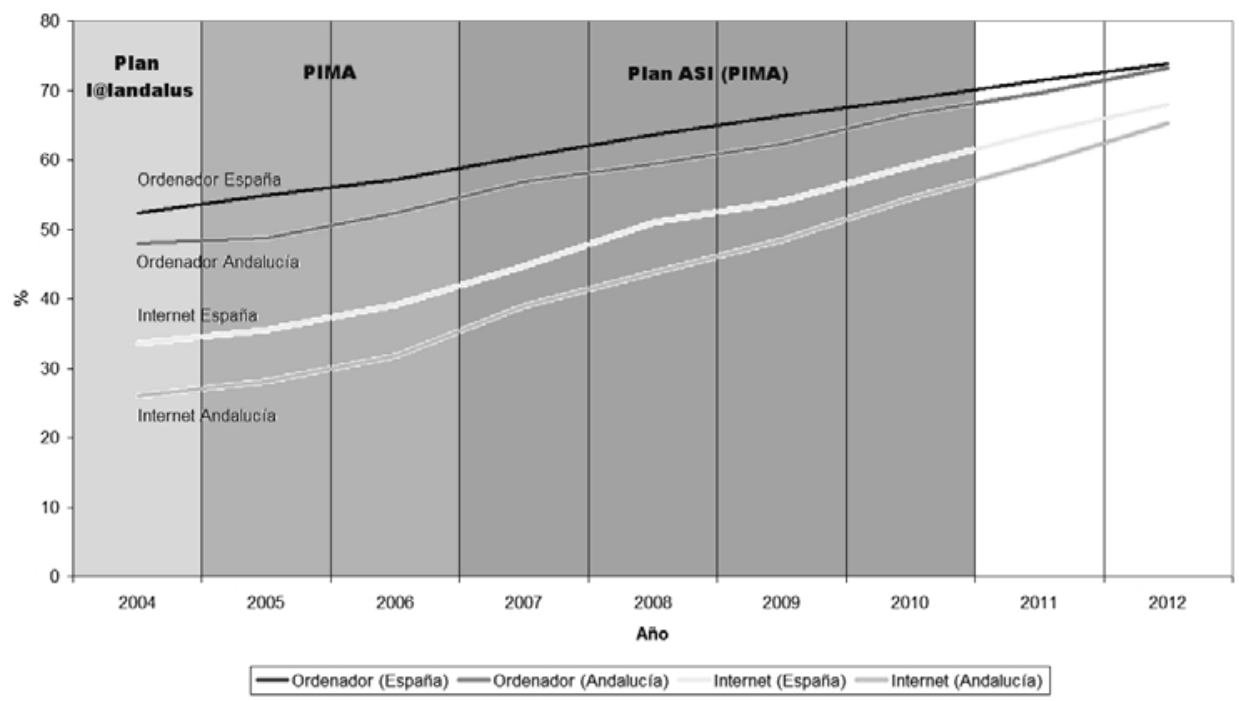

Gráfico 1. Fuente: Elaboración propia a partir de los datos del INE (2012)

c) Disponibilidad de equipamiento e infraestructuras TIC en los hogares andaluces

La disponibilidad de equipamiento y acceso a Internet en función del tamaño del municipio, del número de personas que componen el hogar o de su nivel de ingresos es otro de los aspectos que puede tenerse en cuenta a la hora de analizar el grado de inclusión digital de la población. 
Tabla 3. Fuente: INE (2012)

\begin{tabular}{|c|c|c|c|c|c|}
\hline \multicolumn{6}{|c|}{$\begin{array}{c}\text { Disponibilidad de equipamiento e infraestructuras TIC en los hogares. } \\
\text { Andalucía, } 2012(\%)\end{array}$} \\
\hline & & Ordenador & $\begin{array}{l}\text { Acceso a } \\
\text { Internet }\end{array}$ & $\begin{array}{l}\text { Banda } \\
\text { Ancha }\end{array}$ & Móvil \\
\hline \multirow{5}{*}{$\begin{array}{l}\text { Hábitat: } \\
\text { Número de } \\
\text { habitantes }\end{array}$} & $\begin{array}{l}\text { Más de } 100.000 \text { y } \\
\text { capitales }\end{array}$ & 76,2 & 71,7 & 70,1 & 95,2 \\
\hline & De 50.000 a 100.000 & 81,5 & 70,3 & 69,6 & 98,1 \\
\hline & De 20.000 a 50.000 & 74,8 & 64,7 & 63 & 94,7 \\
\hline & De 10.000 a 20.000 & 70,8 & 63,3 & 59,2 & 96,5 \\
\hline & Menos de 10.000 & 63,8 & 52,7 & 50,4 & 93,3 \\
\hline \multirow{5}{*}{$\begin{array}{l}\text { Tamaño del } \\
\text { hogar: } \\
\text { Número de } \\
\text { personas }\end{array}$} & Una & 42 & 37,9 & 36,7 & 82,2 \\
\hline & Dos & 55,5 & 49,5 & 48,3 & 92,1 \\
\hline & Tres & 82 & 73,2 & 70,6 & 98,8 \\
\hline & Cuatro & 90,5 & 79,8 & 77,4 & 99,5 \\
\hline & Cinco o más & 89,1 & 79,6 & 77 & 100 \\
\hline \multirow{5}{*}{$\begin{array}{l}\text { Ingresos } \\
\text { mensuales } \\
\text { netos del } \\
\text { hogar en } \\
\text { euros }\end{array}$} & Menos de 1.100 & 52,1 & 40 & 38,1 & 90,7 \\
\hline & De 1.100 a 1.800 & 85,3 & 75,5 & 73,9 & 99 \\
\hline & De 1.800 a 2.700 & 90,1 & 87,4 & 86,7 & 100 \\
\hline & Más de 2.700 & 100 & 98,2 & 96,7 & 100 \\
\hline & NS/NR & 76,7 & 70,7 & 67,8 & 95,5 \\
\hline
\end{tabular}

Apreciamos que la disponibilidad de ordenador, Internet y acceso por banda ancha decrece a la par que el tamaño del hábitat (con la excepción del ordenador en los municipios de entre 50.000 y 100.000 habitantes). Estos datos demuestran una brecha territorial en el acceso a las TIC que se hace especialmente evidente en los municipios de menos de 10.000 habitantes (en los que se aprecia un gran salto -incluso de más de 10 puntos porcentuales en el caso del acceso a Internet- con respecto a los que les preceden de manera inmediata, los de 10.000 a 20.000 habitantes).

Otra variable significativa es el número de personas que componen el hogar, ya que a mayor cantidad, se incrementa también la disponibilidad de ordenador, Internet, acceso por banda ancha y teléfono móvil. Existe, por tanto, también una brecha por tamaño del hogar, que se hace especialmente evidente en el caso de los hogares unipersonales.

Otro aspecto clave es el nivel de ingresos del hogar, directamente proporcional a la disponibilidad de equipamiento e infraestructuras TIC en la vivienda. Si consultamos en la encuesta los motivos (no excluyentes) por los que las viviendas principales no disponen de acceso a Internet, en el caso andaluz un 27,2 y 27,5 por ciento, respectivamente, aluden a que los costes del equipo o de la conexión son demasiado elevados. Frente a estos datos, un 62,7 por ciento afirma no necesitar Internet, porque no le resulta útil o interesante, y un 31,2 por ciento disponer de "pocos conocimientos para utilizarlo". La situación económica se convierte, por tanto, en una barrera para la inclusión digital, algo que pone de relieve al menos una cuarta parte de las personas encuestadas que han afirmado no disponer de acceso a Internet en casa. 
d) Uso de las TIC por parte de la ciudadanía andaluza

Tabla 4. Fuente: INE (2012)

\begin{tabular}{|c|c|c|c|c|c|c|c|c|c|c|c|c|c|c|c|c|}
\hline \multicolumn{10}{|c|}{ Uso de las TIC por sexo y edad. Andalucía, 2012 (\%) } \\
\hline $\begin{array}{c}\text { Personas } \\
\text { que, en los } \\
\text { últimos 3 } \\
\text { meses... }\end{array}$ & $\begin{array}{c}\text { Han utilizado el } \\
\text { ordenador }\end{array}$ & \multicolumn{1}{|c|}{$\begin{array}{c}\text { Han utilizado } \\
\text { Internet }\end{array}$} & $\begin{array}{c}\text { Han utilizado } \\
\text { Internet al menos } \\
\text { una vez por } \\
\text { semana }\end{array}$ & $\begin{array}{c}\text { Han comprado a } \\
\text { través de Internet }\end{array}$ & $\begin{array}{c}\text { Han utilizado el } \\
\text { teléfono móvil }\end{array}$ \\
\hline Edad & Mu & Ho & A & Mu & Ho & A & Mu & Ho & A & Mu & Ho & A & Mu & Ho & A \\
\hline $16-24$ & 100 & 90,5 & 95,2 & 99,2 & 91,3 & 95,2 & 94,2 & 88,9 & 91,5 & 11,9 & 30,4 & 21,3 & 100 & 99,5 & 99,7 \\
\hline $25-34$ & 92,1 & 85,7 & 88,8 & 93,4 & 85,3 & 89,3 & 84,5 & 81,6 & 83 & 19,1 & 29 & 24,2 & 98,9 & 100 & 99,5 \\
\hline $35-44$ & 80,8 & 82,5 & 81,7 & 79,5 & 81 & 80,3 & 71,7 & 74 & 72,9 & 20 & 30,9 & 25,6 & 98 & 99,4 & 98,7 \\
\hline $45-54$ & 60 & 65,4 & 62,7 & 55 & 63,1 & 59 & 44,9 & 58,7 & 51,8 & 10,6 & 21,6 & 16,1 & 96,4 & 94,1 & 95,3 \\
\hline $55-64$ & 40,2 & 42,8 & 41,5 & 38,4 & 39,4 & 38,9 & 34 & 36,2 & 35,1 & 5,9 & 8,9 & 7,4 & 89,7 & 87,1 & 88,4 \\
\hline $65-74$ & 6,7 & 13,9 & 10 & 6,3 & 12,5 & 9,2 & 4,4 & 9,6 & 6,8 & 0,8 & 2,6 & 1,6 & 71,9 & 74,7 & 73,2 \\
\hline
\end{tabular}

Analizando los porcentajes por edades, percibimos que existe una diferencia abismal en el uso del ordenador e Internet: del 95,2 por ciento de personas de entre 16 a 24 años que afirman haber utilizado Internet en los tres últimos meses, pasamos al 9,2 por ciento en el caso de las personas de entre 65 y 74 años.

Evaluando los datos por sexo y edad de manera conjunta podemos comprobar además que en la franja de 16 a 34 años, las mujeres superan a los hombres en cuanto a uso de ordenador e Internet. A partir de esa edad se igualan los porcentajes -salvo en la franja de los 45 a los 54, donde el uso por parte de las mujeres es menor-, y es a partir de los 65 años cuando la brecha entre ambos se acrecienta. Sólo el 6,3 por ciento de las mujeres de entre 65 y 74 años reconocen haber usado Internet en los últimos tres meses, frente al 12,5 por ciento de los hombres.

A la luz de los resultados observamos la existencia de una enorme brecha generacional en el uso de las TIC, y de una brecha de género directamente vinculada a ésta, ya que el uso de Internet por parte de las mujeres es superior o prácticamente igual al de los hombres en casi todas las franjas de edad, salvo en las de 45 a 54 años, y 65 a 74 años.

Donde sí que se aprecia una evidente brecha entre mujeres y hombres, independientemente de la franja de edad, es en la compra a través de Internet, donde las mujeres se muestran más reacias (aunque el porcentaje, ya de por sí, sea bastante bajo en ambos sexos).

Otras variables que también resultan de interés a la hora de evaluar la inclusión digital de la población andaluza a través de los usos, son el tamaño de los municipios, el número de personas que componen el hogar, la nacionalidad, el nivel de estudios, la situación laboral y el nivel de ingresos, en todos los casos, desagregado por sexos.

A partir del análisis de estos datos, el primer aspecto que podemos destacar es la existencia de una brecha territorial en el uso de las TIC, marcada por el tamaño de los municipios. A menor tamaño del municipio, menor es el uso del ordenador, de Internet e incluso del móvil. Esto es especialmente patente en el caso de los municipios de menos de 10.000 habitantes, en los que el número de personas que han utilizado Internet en los tres últimos meses es del 59,3 por ciento, frente a 70,2 de las residentes en municipios de más de 100.000 habitantes o capitales de provincia, o el 74,6 por ciento de las que viven en localidades de entre 50.000 y 100.000 . 
Asimismo, se observan diferencias en el uso del ordenador e Internet, en función del tamaño del hogar, siendo bastante menor en el caso de los formados por una única persona (especialmente en el caso de las mujeres).

Por nacionalidades se aprecia un mayor uso por parte de las personas de nacionalidad extranjera. Así, un 72,3 por ciento ha utilizado Internet en los tres últimos meses, frente al 66,9 por ciento de las de nacionalidad española. Aunque las diferencias se acortan cuando hablamos de uso al menos una vez a la semana, donde el porcentaje es del 65,3 frente al 61,3.

Respecto al nivel de estudios, destaca la existencia de una brecha de carácter formativo. Cuanto menor es el nivel, más reducido es el uso que se hace del ordenador e Internet, siendo nulo en el caso de las personas analfabetas. Además, se aprecia una brecha por sexo en los niveles básicos, y un equilibrio a partir de la segunda etapa de Educación Secundaria.

En el caso de la situación laboral, es mayor la utilización de las TIC por parte de las personas ocupadas que de las desempleadas. Destaca el elevado uso por los estudiantes, y el escaso empleo por parte de las personas dedicadas a las labores del hogar. En relación con el sexo, se aprecia una brecha por parte de los hombres en comparación con las mujeres en el caso de los parados $(58,2$ por ciento de hombres frente a 72,2 de mujeres en cuanto a uso de Internet) y pensionistas (49,2 por ciento de hombres frente al 81,5 de mujeres). Por otra parte, es menor el uso del ordenador e Internet entre las personas dedicadas a trabajos manuales, lo que muestra la asociación existente entre el empleo de las TIC y la ocupación.

Por último, el nivel de ingresos también está vinculado con el uso que se hace del ordenador e Internet, o con la compra a través de Internet. A mayor nivel de ingresos, mayores son todos estos porcentajes.

\section{Conclusiones}

Los datos de la encuesta del INE nos permiten distinguir la existencia de una brecha territorial en el acceso a las TIC, ya que Andalucía, pese a las políticas públicas ejecutadas en los últimos años, tanto por parte de la administración central, como de la autonómica, aún sigue registrando porcentajes ligeramente por debajo de la media española. A esta diferencia, se une la relativa al tamaño del municipio, pues un menor número de habitantes va parejo a una menor cantidad de equipamiento y un menor uso de las TIC, lo que se hace especialmente palpable en los municipios de menos de 10.000 habitantes.

Junto a los desniveles territoriales, también encontramos los económicos, ya que las estadísticas demuestran cómo el nivel de ingresos condiciona tanto la disponibilidad de equipamiento como el uso que se hace del ordenador e Internet.

Pero la brecha más evidente de todas es la generacional. Pese a que la encuesta del INE no ofrece los datos de personas por encima de los 74 años (un grave error metodológico, a nuestro entender), la información existente nos alerta de la enorme diferencia tanto en disponibilidad de equipamiento como de uso de las tecnologías entre las personas de mayor y de menor edad. Esto nos lleva a demandar la puesta en marcha de acciones específicas para la inclusión digital de las personas mayores que permitan su total integración en la Sociedad de la Información. 
En el caso de Andalucía, la brecha de género está vinculada directamente con la generacional, así como con un menor nivel de formación. Entre las mujeres más jóvenes, sin embargo, y especialmente en el sector de las desempleadas, apreciamos un alto grado de uso de las TIC, hasta el punto de concluir, a la vista de la fisura existente, que es en el sector de los varones parados donde deben concentrarse, en este caso, las acciones formativas sobre uso de las TIC.

En resumen, podemos concluir que, pese a los esfuerzos realizados -que se reflejan en el progresivo acercamiento a los porcentajes nacionales-, es necesario seguir trabajando por la inclusión digital en Andalucía, concentrando la atención en las personas mayores (y en especial, las mujeres), los habitantes de los municipios de menor población, las personas con un bajo nivel formativo, las dedicadas a las labores del hogar, los pensionistas, los hombres desempleados, así como la población activa ocupada en trabajos de carácter manual, que a diferencia de otros sectores, no tiene la oportunidad de emplear las TIC como parte de su actividad laboral.

\section{Referencias bibliográficas}

AGUSTÍN LACRUZ, María del Carmen y CLAVERO GALOFRÉ, Manuel (2011): "Sociedades digitales y nuevas alfabetizaciones: Políticas públicas de inclusión y alfabetización digital", en CABEZUDO RODRÍGUEZ, Nicolás (Coord.) Inclusión digital: perspectivas y experiencias. Zaragoza, Prensa Universitarias de Zaragoza, pp. 253-274: http://zaguan.unizar.es/record/5993/files/ART-2011-013.pdf [fecha de consulta: 19/10/2012]

BOJA (2003) "Decreto de Medidas de Impulso a la Sociedad del Conocimiento en Andalucía", $\mathrm{n}^{\circ}$ 55, pp. 6024-6034: http://www.juntadeandalucia.es/vgn/images /portal/cit_12354507/31/55/55199535obj.pdf [fecha de consulta: 23/10/2012]

CARIDAD SEBASTIÁN, Mercedes et al. (2006): "Sociedad de la Información e inclusión digital en España: iniciativas y proyectos en marcha. Segunda parte". Ciencias de la Información, vol. 37, $\mathrm{n}^{\mathrm{o}} 2-3$, pp. 15-33. http://redalyc.uaemex.mx/src /inicio/ArtPdfRed.jsp?iCve=181418190002 [fecha de consulta: 20/10/2012]

GOBIERNO DE ESPAÑA (2000): “Info XXI": http://www.internautas.org/documentos/infoxxi.pdf. [fecha de consulta: 21/10/2012]

GOBIERNO DE ESPAÑA (2003): "España.es": http://campus.usal.es/ derinfo/derinfo/Espana.es/espana_es.pdf. [fecha de consulta: 21/10/2012]

GOBIERNO DE ESPAÑA (2005): “Ingenio 2010” https://www.planavanza.es/InformacionGeneral/PlanAvanza1/Antecedentes/bb531fd33c2a4a5e9fe64a7b05159cbb PROGRAMA_INGENIO_2010.pdf. [fecha de consulta: 21/10/2012]

GOBIERNO DE ESPAÑA (2005): "Plan Avanza": https://www.planavanza.es/InformacionGeneral/PlanAvanza1/Descargas/2a392d4f65d9404fb83fc3d2f64eceadplan_avanza_documento_completo.pdf. [fecha de consulta: 21/10/2012]

INE (2012) Encuesta sobre Equipamiento y Uso de Tecnologías de la Información y Comunicación en los hogares. Año 2012: http://www.ine.es/jaxi/menu.do?type =pcaxis\&path=/t25/p450/a2012/\&file=pcaxis. [fecha de consulta: $25 / 10 / 2012$ ] 
JUNTA DE ANDALUCÍA (2002) Plan I@landalus.

JUNTA DE ANDALUCÍA (2006): "Plan Andalucía Sociedad de la Información 20072010 (Plan ASI)": http://www.juntadeandalucia.es/innovacioncienciayempresa/planASIfiles/files/file/Plan_ASI_2007_2010_070423.pdf [fecha de consulta: 23/10/2012]

LÓPEZ LÓPEZ, Pedro y SAMEK, Toni (2009): "Inclusión digital: Un nuevo derecho humano". Educación y Biblioteca, n $^{\circ}$ 172, pp. 114-118: http://dialnet.unirioja.es /descarga/articulo/3026328.pdf. [fecha de consulta: 19/10/2012]

ROBINSON, Scott S. (2005): "Reflexiones sobre la inclusión digital". Nueva Sociedad, $\mathrm{n}^{\mathrm{o}}$ 195, pp. 126-140: http://www.nuso.org/upload/articulos/3244_1.pdf. [fecha de consulta: 19/10/2012]

SOTO PÉREZ, Francisco Javier y FERNÁNDEZ GARCÍA, Juan José (2001): "Los retos de la educación ante la exclusión digital”, en SOTO PÉREZ, F. J. y RODRÍGUEZ VÁZQUEZ, J. (Coord.) Tecnología, Educación y Diversidad: Retos y Realidades de la Inclusión Digital, Murcia, Consejería de Educación y Cultura, pp. 1-5: http://diversidad.murciaeduca.es/tecnoneet/docs/2004/1-62004.pdf [fecha de consulta: 20/10/2012]

UE (2010) Estrategia Europa 2020 para un crecimiento inteligente, sostenible e integrador: http://ec.europa.eu/commission_2010-2014/president/news/documents/pdf/20100303_1_es.pdf. [fecha de consulta: 20/10/2012]

VASAK, Karel (1982): Las dimensiones internacionales de los derechos humanos. París, Unesco.

\section{$M^{a}$ Teresa VERA BALANZA}

Universidad de Málaga

Facultad de Ciencias de la Comunicación

Departamento de Periodismo

Profesora Contratada Doctora acreditada a Titular del Departamento de Periodismo mvb@uma.es

\section{Ma Purificación SUBIRES MANCERA}

Universidad de Málaga

Facultad de Ciencias de la Comunicación

Departamento de Periodismo

Becaria de Formación de Personal Docente e Investigador (Junta de Andalucía) purificacion@uma.es 